\title{
Systemic Corticosteroids in COVID-19: Critical Appraisal of the WHO Meta-analysis
}

\section{Sérgio Renato da Rosa Decker ${ }^{1}$ (i) Eduardo Gehling Bertoldi ${ }^{2}$}

${ }^{1}$ Corresponding author. Federal University of Rio Grande do Sul (Porto Alegre). Rio Grande do Sul, Brazil. sergioodecker@hotmail.com ${ }^{2}$ Federal University of Rio Grande do Sul (Porto Alegre). Rio Grande do Sul, Brazil. eg.bertoldi@gmail.com

KEYWORDS: Meta-Analysis. Corticosteroids. Coronavirus.

\section{Narrative}

Effective therapies for COVID-19 treatment are essential in the context of an ongoing pandemic. Since the publication of the RECOVERY study ${ }^{1}$, dexamethasone $6 \mathrm{mg}$ daily has become routine treatment in many medical services with SARS-Cov-2 infected patients. In the wake of this potentially practice-changing publication, it is imperative to evaluate if other studies with similar approaches would have the same results, if the results show consistency in different clinical scenarios, and if other corticosteroids can achieve the benefit found in the RECOVERY trial with dexamethasone because it is a "class effect." Therefore, the WHO Rapid Evidence Appraisal for COVID-19 Therapies (REACT) Working Group conducted a meta-analysis on steroids and COVID-19?. The authors analyzed seven studies and showed 222 deaths among 678 patients randomized to corticosteroids, and 425 deaths among 1025 patients randomized to usual care or placebo, OR 0.66 (95\% Cl, 0.53-0.82; $\mathrm{P}<0.001$; based on a fixed-effect meta-analysis), with little inconsistency between the trials $(I 2=15.6 \% ; P=$ 0.31 for heterogeneity). They concluded that the meta-analysis showed a benefit of systemic corticosteroids in reducing mortality at 28 days in critically ill patients with COVID-19 than usual care or placebo.

\section{Caveats}

This meta-analysis was a laudable effort of the WHO REACT Working Group and is a useful addition to our knowledge on the use of corticosteroids for COVID-19. However, conclusions should be interpreted with caution, mainly due to two methodological points: a) odds ratio (OR) was used instead of relative risk (RR) as a measure of effect; $b$ ) the conclusion was based on a fixed-effects model. 
Although risk and odds are often used as synonyms when differences between them are ignored, results can be misinterpreted; in randomized clinical trials, differences between odds and risk can become wide ${ }^{3}$. Odds is a poorly understood metric and it is a problem when used to communicate results (for example, explaining an odds of 0.66 as "treatment $X$ was associated with a $44 \%$ reduction in the odds of the outcome") despite some papers discussing odds in terms of risks ${ }^{4}$. However, it is not the only problem: there is inaccuracy when communicating odds ratios like a risk; the same odds ratio could imply a very different relative risk for subgroups because the relative risk also depends on the risk of the outcome in the baseline control group ${ }^{4}$. Therefore, authors of medical research should consider converting odds ratios to relative risk ${ }^{4}$.

When we repeat the analysis using data extracted from Figure 2 of the original study, just by changing the effect's measure to RR (using the software packages R and R Studio) and using fixed-effects model results, we found a summary RR of $0.80(95 \% \mathrm{Cl} 0.70-0.91 ; p<.001)$ regarding mortality in 28 days, when comparing use of systemic corticosteroids and usual care or placebo, contrasting with a summary value with OR of 0.66 . Switching to RR also increased estimated heterogeneity $(1230 \% ; p=0.2)$.

In the same sense, when the authors analyze the data using a fixed-effects model, they are assuming that it refers to a single population, which is presumably not the case, since this meta-analysis included multicenter studies, with different populations (patients from Australia, Brazil, Canada, China, Denmark, France, Ireland, Netherlands, New Zealand, Spain, UK, and US), in different locations/ hospitals and different clinical conditions (mechanical ventilation, admitted to an intensive care unit, intermediate units, among others). Therefore, we believe the most appropriate approach would be to analyze the data using a random-effects model 5 ; when we apply this principle, the difference in mortality at 28 days is not different between systemic corticosteroids and usual care or placebo (OR 0.70; 95\% Cl 0.48-1.01; $\mathrm{p}=0.016$ ).

Finally, if we aggregate the use of a random-effects model and the use of RR as a measure of effect, and estimate heterogeneity by using inverse variance and Paule-Mandel method ${ }^{2}$, we get a RR $0.82(95 \% \mathrm{Cl}, 0.67-1 ; \mathrm{P}<0.05)$, with $30 \%$ heterogeneity $(p=0.2)$; taking into account the impact of this heterogeneity on the confidence interval ${ }^{6}$, we found a wide prediction interval (0.51-1.31; Figure 1).

Figure 1. Meta-analysis of clinical trials with systemic corticosteroids on Critical III COVID-19 patients; summary RR 0.82 (95\% Cl, 0.67-1; P <0.05) with a heterogeneity of $30 \%(p=0.2)$ and prediction interval $0.51-1.31$

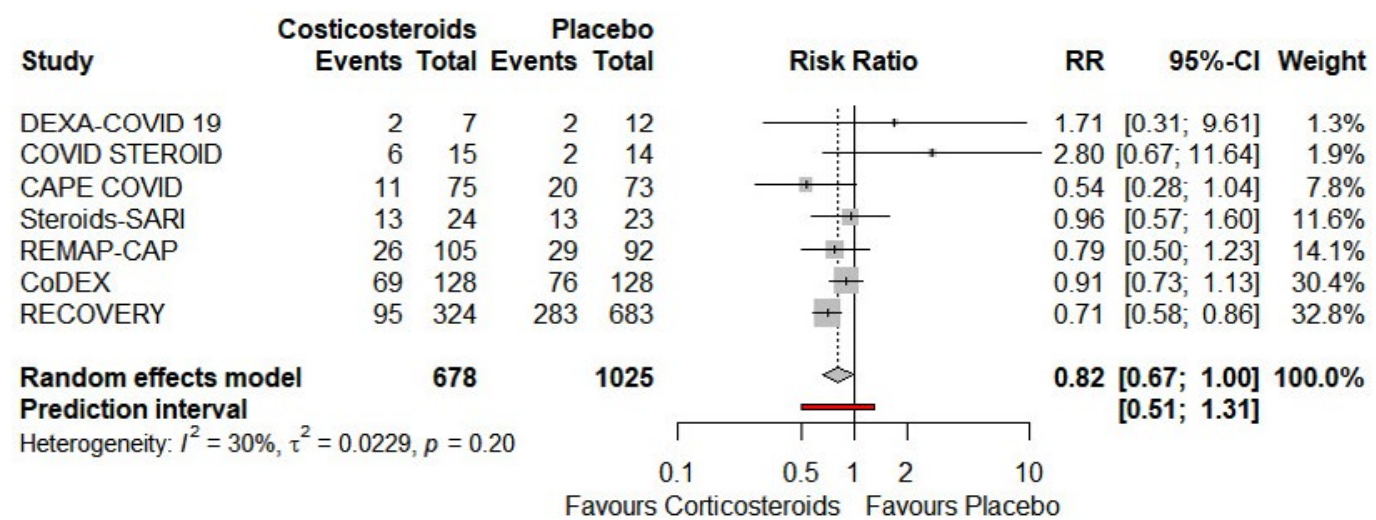


In conclusion, even though the WHO meta-analysis was carried-out promptly, and we are in favor of using dexamethasone in patients infected with SARS-Cov-2 considering the results of the RECOVERY study, the meta-analysis in question is not enough for considering this research topic as definitely concluded, and further studies were warranted to improve our understanding of the role of systemic corticosteroids in COVID-19.

\section{Author contributions}

Decker SRR and Bertoldi, EG designed the critical appraisal. Decker SRR collected the data, analyzed the data, and wrote the manuscript. Bertoldi EG contributed with critical intellectual content and review of the manuscript.

\section{Competing interests}

No financial, legal, or political competing interests with third parties (government, commercial, private foundation, etc.) were disclosed for any aspect of the submitted work (including but not limited to grants, data monitoring board, study design, manuscript preparation, statistical analysis, etc.).

\section{References}

1. RECOVERY Collaborative Group, Horby P, Lim WS, Emberson JR, Mafham M, Bell JL, et al. Dexamethasone in Hospitalized Patients with Covid-19. N Engl J Med. 2021;384(8):693-704. https://doi. org/10.1056/nejmoa2021436

2. WHO Rapid Evidence Appraisal for COVID-19 Therapies (REACT) Working Group, Sterne JAC, Murthy S, Diaz JV, Slutsky AS, Villar J, et al. Association Between Administration of Systemic Corticosteroids and Mortality Among Critically III Patients With COVID-19: A Meta-analysis. JAMA. 2020;324(13):1330-41. https:// doi.org/10.1001/jama.2020.17023

3. Higgins JPT, Thomas J, Chandler J, Cumpston M, Li T, Page MJ, et al, editors. Cochrane Handbook for Systematic Reviews of Interventions. 6th ed. Cochrane; 2019.

4. Grant RL. Converting an odds ratio to a range of plausible relative risks for better comunication of research findings. BMJ. 2014;348:f7450. https://doi.org/10.1136/bmj.f7450

5. Boreinstein M, Hedges LV, Higgins JPT, Rothstein HR. Introduction to Meta-Analysis. New York: Wiley; 2009.

6. IntHout J, loannidis JPA, Rovers MM, Goeman JJ. Plea for routinely presenting prediction intervals in meta-analysis. BMJ Open. 2016;6(7):e010247. http://dx.doi.org/10.1136/ bmjopen-2015-010247 